\title{
THE
}

1984

\section{A Monte Carlo Method for Quantum Boltzmann Statistical Mechanics Using Fourier Representations of Path Integrals}

David L. Freeman

University of Rhode Island, dfreeman@uri.edu

Jimmie D. Doll

Follow this and additional works at: https://digitalcommons.uri.edu/chm_facpubs

Terms of Use

All rights reserved under copyright.

\section{Citation/Publisher Attribution}

Freeman, D. L. \& Doll, J. D. (1984). A Monte Carlo-Method for Quantum-Boltzmann Statistical Mechanics Using Fourier Representations of Path Integrals. J. Chem. Phys., 80, 5709-5718. doi: 10.1063/1.446640 Available at: http://dx.doi.org/10.1063/1.446640 accepted for inclusion in Chemistry Faculty Publications by an authorized administrator of DigitalCommons@URI. For more information, please contact digitalcommons-group@uri.edu. 


\section{AIP $\begin{gathered}\text { mesoumalot } \\ \text { chemical Physics }\end{gathered}$}

A Monte Carlo method for quantum Boltzmann statistical mechanics using Fourier representations of path integrals

David L. Freeman and Jimmie D. Doll

Citation: J. Chem. Phys. 80, 5709 (1984); doi: 10.1063/1.446640

View online: http://dx.doi.org/10.1063/1.446640

View Table of Contents: http://jcp.aip.org/resource/1/JCPSA6/v80/i11

Published by the American Institute of Physics.

Additional information on J. Chem. Phys.

Journal Homepage: http://jcp.aip.org/

Journal Information: http://jcp.aip.org/about/about_the_journal

Top downloads: http://jcp.aip.org/features/most_downloaded

Information for Authors: http://jcp.aip.org/authors

\section{ADVERTISEMENT}
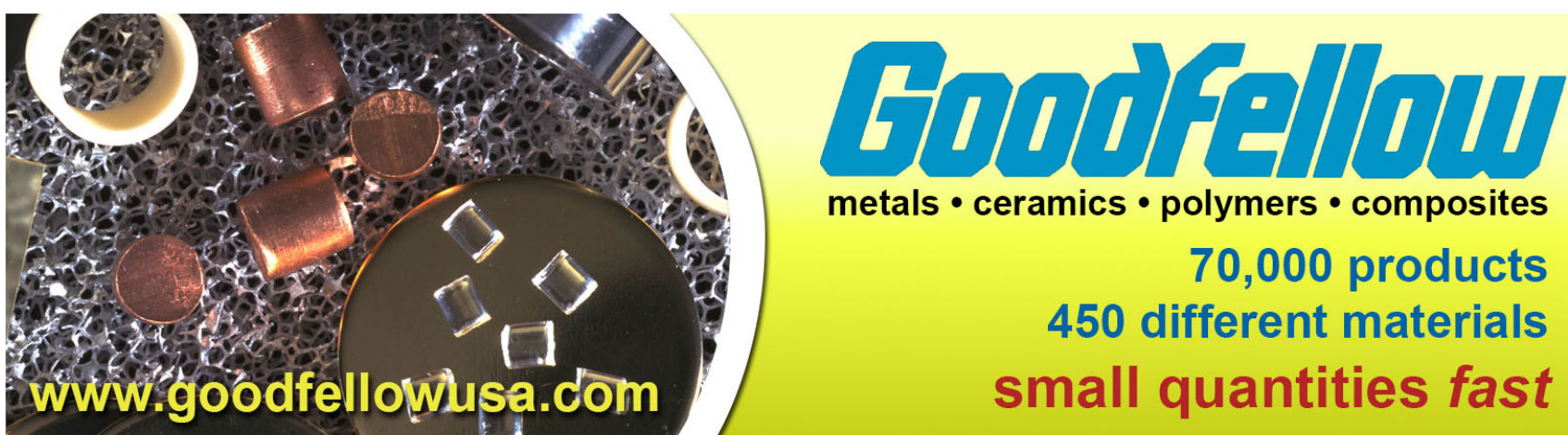
metals • ceramics • polymers $\bullet$ composites

70,000 products 450 different materials small quantities fast 


\title{
A Monte Carlo method for quantum Boltzmann statistical mechanics using Fourier representations of path integrals
}

\author{
David L. Freeman ${ }^{\text {a) }}$ \\ University of California, Los Alamos National Laboratory, Chemistry Division, Los Alamos, New Mexico 87545 \\ and Department of Chemistry, University of Rhode Island, Kingston, Rhode Island 02881 ${ }^{\text {b) }}$ \\ Jimmie D. Doll \\ University of California, Los Alamos National Laboratory, Chemistry Division, MS J569, Los Alamos, \\ New Mexico 87545
}

(Received 14 December 1983; accepted 9 February 1984)

\begin{abstract}
By expanding Feynman path integrals in a Fourier series a practical Monte Carlo method is developed to calculate the thermodynamic properties of interacting systems obeying quantum Boltzmann statistical mechanics. Working expressions are developed to calculate internal energies, heat capacities, and quantum corrections to free energies. The method is applied to the harmonic oscillator, a double-well potential, and clusters of Lennard-Jones atoms parametrized to mimic the behavior of argon. The expansion of the path integrals in a Fourier series is found to be rapidly convergent and the computational effort for quantum calculations is found to be within an order of magnitude of the corresponding classical calculations. Unlike other related methods no special techniques are required to handle systems with strong short-range repulsive forces.
\end{abstract}

\section{INTRODUCTION}

Monte Carlo simulations ${ }^{1}$ provide an extremely valuable method of determining the thermodynamic properties of complex interacting many-body systems. At best, when realistic intermolecular forces are used, Monte Carlo calculations provide information which can be compared with experimental results or used to supplant results for which experiments are unavailable. At worst, when the intermolecular forces used are qualitative, Monte Carlo results provide a benchmark useful for comparison with analytic theories. A significant amount of the current microscopic understanding of the behavior of liquids has evolved directly or indirectly from Monte Carlo simulations. The importance of the technique to modern statistical mechanics cannot be overemphasized.

Until very recently Monte Carlo calculations have been limited to systems for which a classical mechanical description was adequate. Systems with significant contributions from low temperature vibrational motion, tunneling, particle statistics or other inherently quantum effects have been qualitatively treated.

To introduce quantum effects into statistical mechanical treatments of interacting many-body systems there have been a number of recent methods ${ }^{2-8}$ developed which use Feynman path integral ${ }^{9}$ representations of the quantum statistical mechanical density matrix. In most of these applications ${ }^{2-5,7}$ the path integrals have been discretized and short interval approximations have been introduced. Using the short interval approximation, path integral methods have been successfully applied to a number of systems. It has been noted $^{2}$ that systems with strongly repulsive short range interactions (such as Lennard-Jones forces) are poorly treated in the short interval approximation. This limitation has been overcome by a modified short interval formulation given by

a) Visiting Staff Member at Los Alamos National Laboratory, 1982-1983. ${ }^{b)}$ Permanent address.
Barker ${ }^{2}$ for hard core systems. For Lennard-Jones systems with a small finite number of quantum degrees of freedom a numerical matrix multiplication scheme $e^{7}$ has successfully been applied to the calculation of the particle density. We are unaware of any method which has been used to treat systems simultaneously having a large number of quantum degrees of freedom and potentials with strong repulsive cores. For such general systems the convergence difficulties associated with short interval approximations to path integrals imply a need to find alternative approaches to quantum statistical mechanics.

In a recent note ${ }^{8}$ we reported a Monte Carlo approach to quantum Boltzmann statistical mechanics which utilized an alternative method of evaluating the required Feynman path integrals. In this method the paths were expanded in a Fourier series about a fixed path, and the integration over all paths was evaluated by an equivalent integration over Fourier coefficients. We showed that the integration over the Fourier coefficients could be evaluated by the Monte Carlo method introduced by Metropolis et al. ${ }^{10}$ Because the expansion of paths in a Fourier series was found to converge rapidly we indicated that the computational effort in our approach was within an order of magnitude of the corresponding classical calculation. Furthermore in mixed systems with both quantum and classical degrees of freedom advantage could be taken by treating part of the problem classically.

The purposes of the present work are threefold. First, we wish to show that the Fourier method can be applied to systems with repulsive potentials, like Lennard-Jones systems, without modification. Second, we wish to give a description of the method in more detail than was possible in a short note. Finally, we wish to show that the method can be applied to realistic interacting many-body systems. The contents of the remainder of this paper are as follows. In Sec. II we develop the expressions for the quantum statistical me- 
chanical density matrix in a Fourier representation of the required path integrals. We also show how the thermodynamic properties of an interacting quantum system can be evaluated with Monte Carlo methods. In Sec. III we apply the method developed in Sec. II to calculate the internal energy, the free energy and the heat capacity of the linear harmonic oscillator, a double-well potential and clusters of Lennard-Jones representations of argon atoms. In Sec. IV we summarize our conclusions.

\section{THEORY}

In this section we develop the expressions required for Monte Carlo evaluations of the thermodynamic properties of interacting quantum many-body systems. Although some of the expressions developed in this section are available elsewhere $^{8}$ we present sufficient detail to make our approach as clear as possible. The development is limited to Boltzmann statistics.

We consider a general $N$-particle system whose Hamiltonian is given by

$$
\widehat{H}=\sum_{i=1}^{N} \hat{p}_{i}^{2} / 2 m_{i}+V\left(\mathbf{r}_{1}, \ldots, \mathbf{r}_{N}\right)
$$

In Eg. (1) $r_{i}$ is the coordinate of particle $i$ whose mass is $m_{i}$ and $\hat{p}_{i}$ is the momentum operator for particle $i$. From Eq. (1) we define the density operator by

$$
\hat{\rho}=\exp \{-\beta \hat{H}\} \text {, }
$$

where $\beta=1 / k_{B} T, k_{B}$ being the Boltzmann constant and $T$ the absolute temperature. All equilibrium thermodynamic properties of a system can be evaluated in terms of $\hat{\rho}$. For example, the internal energy $\langle U\rangle$ is given by

$$
\langle U\rangle=\frac{\operatorname{Tr} \hat{\rho} \hat{H}}{\operatorname{Tr} \hat{\rho}} .
$$

For Monte Carlo calculations it is convenient to evaluate the traces in Eq. (3) in coordinate representation. Consequently we need to evaluate nondiagonal elements of the density operator. We then write for the density matrix elements

$$
\rho\left(\mathbf{r}_{1}, \ldots, \mathbf{r}_{N}, \mathbf{r}_{1}^{\prime}, \ldots, \mathbf{r}_{N}^{\prime}\right)=\left\langle\mathbf{r}_{1}, \ldots, \mathbf{r}_{N}|\hat{\rho}| \mathbf{r}_{i}^{\prime}, \ldots, \mathbf{r}_{N}^{\prime}\right\rangle
$$

Density matrix elements are often evaluated numerically by discretizing integral representations of Eq. (4) and using short interval approximations. ${ }^{2-5,7}$ To allow for comparisons with the method we introduce shortly we now briefly review this approach. For convenience we let $R$ be the collective $3 N$ dimensional coordinate of our many body system. Then we can write

$$
\begin{aligned}
\rho\left(\mathbf{R} ; \mathbf{R}^{\prime}\right\rangle= & \left\langle\mathbf{R}\left|\left(e^{-\beta \hat{H} / M}\right)^{M}\right| \mathbf{R}^{\prime}\right\rangle \\
= & \int d^{3 N} R_{1} d^{3 N} R_{2} \cdots d^{3 N} R_{M}\langle\mathbf{R}| e^{-\beta H / M} \\
& \times\left|\mathbf{R}_{1}\right\rangle\left\langle\mathbf{R}_{1}\left|e^{-\beta \hat{H} / M}\right| \mathbf{R}_{2}\right\rangle \cdots\left\langle\mathbf{R}_{M}\left|e^{-\beta \hat{H} / M}\right| \mathbf{R}^{\prime}\right\rangle .(6)
\end{aligned}
$$

For large $M$ each factor in Eq. (6) can be replaced by the approximation $^{2}$

$$
\begin{aligned}
& \left\langle\mathbf{R}\left|e^{-\beta \hat{H} / M}\right| \mathbf{R}^{\prime}\right\rangle \cong\left(\frac{4 \pi}{M} \frac{\hbar^{2}}{2 m} \beta\right)^{-3 N / 2} \\
& \quad \times \exp \left[-M \frac{\left(\mathbf{R}-\mathbf{R}^{\prime}\right)^{2} m}{2 \hbar^{2} \beta}-\frac{\beta}{2 M}\left(V(\mathbf{R})+V\left(\mathbf{R}^{\prime}\right)\right\}\right],
\end{aligned}
$$

which becomes exact as $M$ becomes infinite. As Barker ${ }^{2}$ has stated the approximation given in Eq. (7) will be accurate as long as the potential energy function $V$ changes slowly compared to the first term in the exponential. For systems with strongly repulsive short range interactions (e.g., LennardJones systems) the potential energy may vary too rapidly for Eq. (6) to be well converged. Consequently to treat the important class of systems with strongly repulsive cores it is important to seek alternative approaches for evaluating the quantum mechanical density matrix which may be more rapidly convergent.

The approach to the evaluation of the quantum density matrix used in this work begins with the limiting form of Eq. (6) when $M$ becomes infinite. In this continuous limit the density matrix is written in path integral form ${ }^{9}$

$$
\begin{aligned}
\rho\left(\mathbf{r}_{1}^{\prime}, \ldots, \mathbf{r}_{N}^{\prime} ; \mathbf{r}_{1}, \ldots, \mathbf{r}_{N}\right) \\
=\int \mathscr{D} \mathbf{r}_{1}(u) \mathscr{D} \mathbf{r}_{2}(u) \ldots \mathscr{D} \mathbf{r}_{N}(u) \exp \left\{-\frac{1}{\hbar} \int_{0}^{\beta \hbar}\right. \\
\left.\quad \times d u\left[\frac{1}{2} \sum_{i=1}^{N} m_{i} \dot{\mathbf{r}}_{i}(u)^{2}+V\left[\mathbf{r}_{1}(u), \ldots, \mathbf{r}_{N}(u)\right]\right]\right\}
\end{aligned}
$$

In Eq. (8) the path integrals are evaluated over all paths which connect $r_{1}$ to $r_{1}^{\prime}, r_{2}$ to $r_{2}^{\prime}, \ldots$, and $r_{N}$ to $r_{N}^{\prime}$. We transform each path integral to an ordinary Riemann integral by using Feynman's trick ${ }^{9}$ of expanding the path in a Fourier series about a fixed path which connects to $\mathbf{r}_{i}$ to $\mathbf{r}_{i}^{\prime}$. The exact nature of the fixed path is arbitrary and can be chosen to optimize the computational work required for a particular problem. For a wide variety of situations we have found it convenient to choose a linear fixed path connecting $\mathbf{r}_{i}$ to $\mathbf{r}_{i}^{\prime}$. For this choice we write for the $x$ component of coordinate $\mathbf{r}_{i}$ :

$$
x_{i}(u)=x_{i}+\left(x_{i}^{*}-x_{i}\right) u / \beta \hbar+\sum_{k=1}^{\infty} a_{k x i} \sin \frac{k \pi u}{\beta \hbar} .
$$

Another possible choice of fixed path will be given in the Appendix for systems whose motion is primarily vibrational. If we introduce Eq. (9) into Eq. (8) the $u$ integration over the kinetic energy term in the exponential can be evaluated analytically. The resulting path integrals are replaced by integrals over all Fourier components and we obtain

$$
\begin{aligned}
& \rho\left(\mathbf{r}_{1}^{\prime}, \ldots, \mathbf{r}_{N}^{\prime} ; \mathbf{r}_{1}, \ldots, \mathbf{r}_{N}\right)=J \prod_{i=1}^{N} \exp \left\{-\frac{m_{i}}{2 \beta \hbar^{2}}\left(\mathbf{r}_{i}-\mathbf{r}_{i}^{\prime}\right)^{2}\right\} \\
& \quad \times \prod_{k=1}^{\infty} \int d^{3} a_{k i} \exp \left\{-a_{k i}^{2} / 2 \sigma_{k i}^{2}\right\} \\
& \quad \times \exp \left\{-\frac{1}{\hbar} \int_{0}^{\beta \hbar} V\left[\mathbf{r}_{1}(u), \ldots, \mathbf{r}_{N}(u)\right] d u\right\} .
\end{aligned}
$$

In Eq. (10) $J$ is the Jacobian of the transformation from the integral over all paths to the integral over all Fourier coefficients. This Jacobian has been evaluated by Miller ${ }^{11}$ in another context who found

$$
J=\prod_{i=1}^{N}\left(\frac{m_{i}}{2 \pi \hbar^{2} \beta}\right)^{3 / 2} \prod_{k=1}^{\infty} \frac{1}{\sqrt{2 \pi} \sigma_{k i}}
$$

In this work our only concern is the fact that $J$ is a constant; i.e., independent of $r_{i}$ and $V$. In Eqs. (10) and (11) $\sigma_{k i}$ is given by 


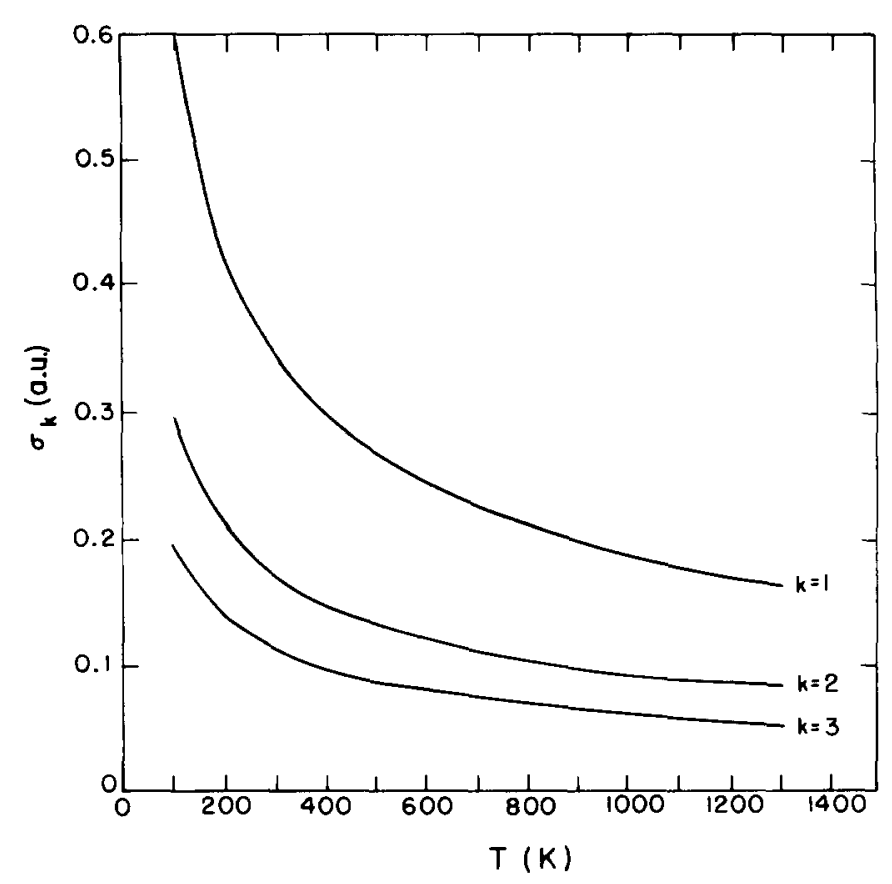

FIG. 1. The width of the Gaussian distribution of Fourier coefficients as a function of temperature and Fourier index ( $m=$ proton mass.)

$$
\sigma_{k i}=\left[2 \beta \hbar^{2} / m_{i}(\pi k)^{2}\right]^{1 / 2},
$$

The $\mathbf{a}_{k i}$ Fourier coefficients appear in Eq. (10) both inside the potential energy function and with a Gaussian dependence. The width of the Gaussian factors increases with decreasing temperature so that the extent of quantum behavior is related to the magnitude of $\sigma_{k i}$. This can be understood by examining Fig. 1 where $\sigma_{k i}$ is plotted against the temperature for $k=1,2$, and 3. At low temperature $\sigma_{k i}$ is seen to be large whereas $\sigma_{k i}$ approaches zero at high temperatures. Using the relation

$$
\delta(x)=\lim _{\sigma \rightarrow 0} \frac{1}{\sqrt{2 \pi} \sigma} \exp \left\{-x^{2} / 2 \sigma^{2}\right\},
$$

and Eqs. (10) and (11) it is clear that

$$
\begin{aligned}
\lim _{\beta \rightarrow 0} \rho\left(\mathbf{r}_{1}, \ldots, \mathbf{r}_{N} ; \mathbf{r}_{1}^{\prime}, \ldots, \mathbf{r}_{N}^{\prime}\right) \\
=\left(\frac{2 \pi m}{h^{2} \beta}\right)^{3 N / 2} \exp \left\{-\beta V\left(\mathbf{r}_{1}, \ldots, \mathbf{r}_{N}\right)\right\},
\end{aligned}
$$

which is the classical density.

From Eq. (10) we now develop two alternative expressions for evaluating the internal energy. The first expression is derived by direct evaluation of $\mathrm{Eq}$. (3). The second expression uses the quantum mechanical virial theorem. From Eq. (3)

$$
\begin{aligned}
\langle U\rangle=\int & d^{3} r_{1} \ldots d^{3} r_{N} \rho\left(\mathbf{r}_{1}, \ldots, \mathbf{r}_{N} ; \mathbf{r}_{1}^{\prime}, \ldots, \mathbf{r}_{N}^{\prime}\right) \\
& \times\left[-\frac{\hbar^{2}}{2} \sum_{i} \frac{1}{m_{i}} \nabla_{i}^{2}+V\left(\mathbf{r}_{1}, \ldots, \mathbf{r}_{N}\right)\right] \begin{array}{c}
\mathbf{r}_{1}=\mathbf{r}_{1}^{\prime} \\
\vdots \\
\mathbf{r}_{N}=\mathbf{r}_{N}^{\prime}
\end{array} \\
& \int d^{3} r_{1} \ldots d^{3} r_{N} \rho\left(\mathbf{r}_{1}, \ldots, \mathbf{r}_{N} ; \mathbf{r}_{1}, \ldots, \mathbf{r}_{N}\right) .
\end{aligned}
$$

We introduce Eq. (10) into Eq. (15) and truncate the product on $k$ in Eq. (10) at an upper limit, $k_{\text {max }}$. The derivatives can be evaluated analytically and we arrive at the expression

$$
\begin{aligned}
\langle\hat{H}\rangle_{k_{\max }}= & \frac{3 N}{2 \beta}+\int d^{3} r_{1} \ldots d^{3} r_{N} \int \prod_{k=1}^{k_{\max }} d^{3} a_{k 1} \ldots d^{3} a_{k N} \\
& \times \exp \left\{-\sum_{i=1}^{N} a_{k i}^{2} / 2 \sigma_{k i}^{2}\right\} \exp \left\{-\frac{1}{\hbar} \int_{0}^{\beta \hbar} \mathrm{V}\left[\mathbf{r}_{1}(u), \ldots, \mathbf{r}_{N}(u)\right] d u\right\} \\
& \times\left(V\left(\mathbf{r}_{1}, \ldots, \mathbf{r}_{N}\right)-\frac{\hbar^{2}}{2} \sum_{i=1}^{N} \frac{1}{m_{i}}\left(\left[\frac{1}{\hbar} \int_{0}^{\beta \hbar}\left(1-\frac{u}{\beta \hbar}\right) \hat{r}_{i} \cdot \nabla_{i} V\left(\mathbf{r}_{1}(u), \ldots, \mathbf{r}_{N}(u)\right) d u\right]^{2}\right.\right. \\
& \left.\left.-\frac{1}{\hbar} \int_{0}^{\beta \hbar}\left(1-\frac{u}{\beta \hbar}\right)^{2} \nabla_{i}^{2} V\left(\mathbf{r}_{1}(u), \ldots, \mathbf{r}_{N}(u)\right) d u\right)\right) / \int d^{3} r_{1} \cdots d^{3} r_{N} \int \prod_{k=1}^{k_{\max }} d^{3} a_{k 1} \cdots d^{3} a_{k N} \\
& \times \exp \left\{-\sum_{i=1}^{N} a_{k i}^{2} / 2 \sigma_{k i}^{2}\right\} \exp \left\{-\frac{1}{\hbar} \int_{0}^{\beta \hbar} V\left[\mathbf{r}_{1}(u), \ldots, \mathbf{r}_{N}(u)\right] d u\right\},
\end{aligned}
$$

where

$$
\lim _{k_{\max } \rightarrow \infty}\langle H\rangle_{k_{\max }}=\langle U\rangle
$$

Equation (16) can be evaluated using the method of Metropolis et al. ${ }^{10}$ for the integrations over $\mathbf{r}_{1}, \ldots, \mathbf{r}_{N}$ and $\mathbf{a}_{k 1}, \ldots, \mathbf{a}_{k N}$. The weight function contains a one-dimensional integration over $u$ which can be evaluated with Gauss quadrature. Gauss quadrature can also be used to evaluate the additional $u$ integrations in Eq. (16). The utility of Eq. (16) depends upon the rapidity with which Eq. (17) is satisfied for finite $k_{\max }$, and the ease in performing the $u$ integrations by Gauss quadrature. As we shall see in the next section for all cases we have studied $\langle\hat{H}\rangle_{k_{\max }}$ differs from $\langle U\rangle$ negligibly when only a few Fourier coefficients are included. 
As an alternative to Eq. (16) the internal energy can be evaluated using the quantal virial theorem which states that

$$
\frac{\operatorname{Tr} \hat{\rho} \hat{T}}{\operatorname{Tr} \hat{\rho}}=\operatorname{Tr} \hat{\rho}\left[\frac{1}{2} \sum_{i=1}^{N} \mathbf{r}_{i} \cdot \nabla_{i} V\right] / \operatorname{Tr} \hat{\rho},
$$

where $\hat{T}$ is the kinetic energy operator. Equation (18) is valid provided that the density matrix elements used are exact. If the products on $k$ in Eq. (10) are truncated at an upper limit, $k_{\max }$ we then can write

$$
\begin{aligned}
\langle\hat{\epsilon}\rangle_{k_{\max }}= & \int d^{3} r_{1} \cdots d^{3} r_{N} \int \prod_{k=1}^{k_{\max }} d^{3} a_{k 1} \cdots d^{3} a_{k N} \exp \left\{-\sum_{i=1}^{N} a_{k i}^{2} / 2 \sigma_{k i}^{2}\right\} \exp \left\{-\frac{1}{\hbar} \int_{0}^{\beta \hbar} V\left[\mathbf{r}_{1}(u), \ldots, \mathbf{r}_{N}(u)\right] d u\right\} \\
& \times \hat{\epsilon}\left(\mathbf{r}_{1}, \ldots, \mathbf{r}_{N}\right) / \int d^{3} r_{1} \cdots d^{3} r_{N} \int_{k=1}^{k_{\max }} d^{3} a_{k 1} \cdots d^{3} a_{k N} \exp \left\{-\sum_{i=1}^{N} a_{k i}^{2} / 2 \sigma_{k i}^{2}\right\} \exp \left\{-\frac{1}{\hbar} \int_{0}^{\beta \hbar} V\left[\mathbf{r}_{1}(u), \ldots, \mathbf{r}_{N}(u)\right] d u\right\},
\end{aligned}
$$

where

$$
\hat{\boldsymbol{\epsilon}}\left(\mathbf{r}_{1}, \ldots, \mathbf{r}_{N}\right)=V\left(\mathbf{r}_{1}, \ldots, \mathbf{r}_{N}\right)+\frac{1}{2} \sum_{i=1}^{N} \mathbf{r}_{i} \cdot \nabla_{i} V .
$$

From the virial theorem we know that

$$
\lim _{k_{\max } \rightarrow \infty}\langle\hat{\epsilon}\rangle_{k_{\max }}=\langle U\rangle
$$

Although Eqs. (17) and (21) look similar it is important to recognize that in general

$$
\langle\widehat{H}\rangle_{k_{\max }} \neq\langle\widehat{\epsilon}\rangle_{k_{\max }} \text {, }
$$

and the convergence properties of Eqs. (17) and (21) may not be the same. Equation (19) is numerically easier to evaluate than Eq. (16), but as we shall see in the next section Eq. (16) often converges much more rapidly than Eq. (19) with respect to $k_{\text {max }}$. Consequently Eq. (16) is the preferred expression in many cases.

The constant volume heat capacity $C_{V}$ defined by

$$
C_{V}=-\frac{1}{k_{B} T^{2}} \frac{\partial\langle U\rangle}{\partial \beta}
$$

can be evaluated numerically either by finite difference methods on calculated internal energies or by direct differentiation of $\mathrm{Eq}$. (16) or Eq. (19) with respect to $\beta$. For example in those cases for which Eq. (19) is well converged differentiation with respect to $\beta$ gives the expression

$$
\begin{aligned}
C_{V}= & \frac{1}{k_{B} T^{2}} \int d^{3} r_{1} \cdots d^{3} r_{N} \int \prod_{k=1}^{\infty} d^{3} a_{k 1} \cdots d^{3} a_{k N} \exp \left\{-\sum_{i=1}^{N} a_{k i}^{2} / 2 \sigma_{k i}^{2}\right\} \exp \left\{-\frac{1}{\hbar} \int_{0}^{\beta \hbar} V\left[\mathbf{r}_{1}(u), \ldots, \mathbf{r}_{N}(u)\right] d u\right\} \\
& \times\left[\hat{\epsilon}\left(\mathbf{r}_{1}, \ldots, \mathbf{r}_{N}\right)-\langle U\rangle\right]\left[\frac{1}{\beta} \sum_{k=1}^{\infty} \sum_{i=1}^{N} a_{k i}^{2} / 2 \sigma_{k i}^{2}-V\left(\mathbf{r}_{1}, \ldots, \mathbf{r}_{N}\right)\right]+\frac{1}{\hbar} \int_{0}^{\beta \hbar} d u \sum_{i=1}^{N} \\
& \left.\times\left(\frac{\partial V}{\partial x_{i}} \sum_{k=1}^{\infty} a_{k x i} \frac{k \pi u}{\beta \hbar} \cos \frac{k \pi u}{\beta \hbar}+\frac{\partial V}{\partial y_{i}} \sum_{k=1}^{\infty} a_{k y i} \frac{k \pi u}{\beta \hbar} \cos \frac{k \pi u}{\beta \hbar}+\frac{\partial V}{\partial z_{i}} \sum_{k=1}^{\infty} a_{k z i} \frac{k \pi u}{\beta \hbar} \cos \frac{k \pi u}{\beta \hbar}\right)\right] / \\
& \int d^{3} r_{1} \cdots d^{3} r_{N} \int \prod_{k=1}^{\infty} d^{3} a_{k 1} \cdots d^{3} a_{k N} \exp \left\{-\sum_{i=1}^{N} a_{k i}^{2} / 2 \sigma_{k i}^{2}\right\} \exp \left\{-\frac{1}{\hbar} \int_{0}^{\beta \hbar} V\left[\mathbf{r}_{1}(u), \ldots, \mathbf{r}_{N}(u)\right] d u\right\} .
\end{aligned}
$$

Equation (24) can be evaluated by the methods of Metropolis et al. ${ }^{10}$ with Monte Carlo evaluations of the integrations on the coordinates and Fourier coefficients, and Gauss techniques can be applied to the one-dimensional $u$ integrations. Whether finite difference techniques or direct evaluation of Eq. (24) is to be preferred is a function of the system studied. In those cases we have investigated, finite difference techniques have proved to be satisfactory.

A number of techniques have been developed ${ }^{12}$ to evaluate the free energy of an interacting many-particle system using classical Monte Carlo methods. Many of these classical techniques can be applied without change in the quantum case and we make no effort to review the extensive literature on the subject here. When classical free energies are available quantum corrections can be included by calculating the ratio of the quantum-mechanical partition function $Z_{\mathrm{QM}}$ to the classical partition function $Z_{\mathrm{CM}}$. We define 


$$
\Delta=Z_{\mathrm{QM}} / Z_{\mathrm{CM}} .
$$

From the fact that

$$
A=-k T \ln Z_{\mathrm{QM}}
$$

we can write

$$
\begin{aligned}
A & =-k T \ln \left(Z_{\mathrm{CM}} \Delta\right) \\
& =A_{\mathrm{CM}}-k T \ln \Delta,
\end{aligned}
$$

where $A_{\mathrm{CM}}$ is the classical Helmholtz free energy. The ratio $\Delta$ is also useful in calculating quantum corrections to the internal energy because

$$
\langle U\rangle=-\left(\partial \ln Z_{\mathrm{QM}} / \partial \beta\right)_{V},
$$

so that

$$
\langle U\rangle=\langle U\rangle_{\mathrm{CM}}-\left(\frac{\partial \ln \Delta}{\partial \beta}\right)_{V},
$$

where $\langle U\rangle_{\mathrm{CM}}$ is the classical internal energy. To evaluate Eq. (25) with Metropolis-Monte Carlo methods we use Eq. (10) and obtain

$$
\begin{aligned}
\Delta= & \int d^{3} r_{1} \ldots d^{3} r_{N} \int \prod_{k=1}^{\infty} d^{3} a_{k 1} \ldots d^{3} a_{k N} \exp \left\{-\sum_{i=1}^{N} a_{k i}^{2} / 2 \sigma_{k i}^{2}\right\} \exp \left\{-\frac{1}{\hbar} \int_{0}^{\beta \hbar} V\left[\mathbf{r}_{1}(u), \ldots, \mathbf{r}_{N}(u)\right] d u\right\} \\
& \int d^{3} r_{1} \cdots d^{3} r_{N} \int \prod_{k=1}^{\infty} d^{3} a_{k 1} \ldots d^{3} a_{k N} \exp \left\{-\sum_{i=1}^{N} a_{k i}^{2} / 2 \sigma_{k i}^{2}\right\} \exp \left\{-\beta V_{\mathrm{CM}}\left(\mathbf{r}_{1}, \ldots, \mathbf{r}_{N}\right)\right. \\
= & \int d^{3} r_{1} \ldots d^{3} r_{N} \int \prod_{k=1}^{\infty} d^{3} a_{k 1} \ldots d^{3} a_{k N} \exp \left\{-\sum_{i=1}^{N} a_{k i}^{2} / 2 \sigma_{k i}^{2}\right\} \exp \left\{-\beta V_{\mathrm{CM}}\left(\mathbf{r}_{1}, \ldots, \mathbf{r}_{N}\right)\right\} \\
& \times \exp \left\{-\frac{1}{\hbar} \int_{0}^{\beta \hbar}\left(V\left[\mathbf{r}_{1}(u), \ldots, \mathbf{r}_{N}(u)\right]-V_{\mathrm{CM}}\left(\mathbf{r}_{1}, \ldots, \mathbf{r}_{N}\right)\right) d u\right\} / \int d^{3} r_{1} \ldots d^{3} r_{N} \int \prod_{k=1}^{\infty} d^{3} a_{k 1} \ldots d^{3} a_{k N} \\
& \times \exp \left\{-\sum_{i=1}^{N} a_{k i}^{2} / 2 \sigma_{k i}^{2}\right\} \exp \left\{-\beta V_{\mathrm{CM}}\left(\mathbf{r}_{1}, \ldots, \mathbf{r}_{N}\right)\right\} .
\end{aligned}
$$

In Eq. (32) we sample over the bare classical potential $V_{\mathrm{CM}}\left(\mathbf{r}_{1}, \ldots, \mathbf{r}_{N}\right)$.

\section{APPLICATIONS}

In this section we illustrate the formalism developed in $\mathrm{Sec}$. II by example calculations on a number of systems. The principal conclusions of this section are:

(i) the expansion of path integrals in a Fourier series about a straight line path converges very rapidly for a wide variety of physical systems over a wide temperature range;

(ii) the one-dimensional $u$ integrations required for the evaluation of thermodynamic properties are easily evaluated with Gauss-Legendre quadrature using a small number of Gauss points; and

(iii) the method has no special difficulties when applied to systems with strong short range repulsions. Some of the results presented here were included in the short note published previously. ${ }^{8}$ We repeat them to make the present work self-contained.

\section{A. The one-dimensional harmonic oscillator}

The one-dimensional harmonic oscillator is analytically solvable, and many chemical problems involve vibrational motion. Consequently in treating the harmonic oscillator by our numerical methods we will illustrate many of the features to be expected for more complex systems. Analytically the internal energy of the linear harmonic oscillator is given by the well known expression

$$
\langle U\rangle=\frac{\hbar \omega}{2}+\frac{\hbar \omega \exp \{-\beta \hbar \omega)\}}{1-\exp \{-\beta \hbar \omega\}},
$$

where $\omega$ is the oscillator frequency. We can also calculate the internal energy of the oscillator either from Eq. (16) or Eq. (19). Because the virial theorem for the harmonic oscillator takes the form

$$
\langle\hat{T}\rangle=\langle V\rangle,
$$

and $\langle V\rangle$ is required for Eq. (16), it is clear that Eq. (16) cannot converge any faster than Eq. (19) with respect to the number of Fourier components included. Consequently we only examine Eq. (19) for the oscillator. In Eq. (19) all integrations are Gaussian and can be evaluated analytically. The result is

$$
\langle\hat{\epsilon}\rangle_{k_{\max }}=\frac{1}{\beta-2 \beta \sum_{k=1}^{k_{\max }} B_{k}},
$$

where

$$
B_{k}=(\beta \hbar \omega)^{2} \frac{\left[1-(-1)^{k}\right]^{2}}{(k \pi)^{2}\left[(k \pi)^{2}+(\beta \hbar \omega)^{2}\right]} .
$$


TABLE I. The energy of the harmonic oscillator as a function of the number of Fourier coefficients [Eq. (35)].

\begin{tabular}{lcc}
\hline \hline$\beta \hbar \omega$ & $k_{\max }$ & $\beta\langle\hat{\boldsymbol{\epsilon}}\rangle_{k_{\max }}$ \\
\hline 10.0 & 1 & 3.813 \\
& 3 & 4.661 \\
& 5 & 4.873 \\
& 7 & 4.942 \\
& 9 & 4.969 \\
& 11 & 4.982 \\
& 13 & 4.989 \\
& 15 & 4.992 \\
5.0 & $\infty$ & 5.001 \\
& 1 & 2.387 \\
& 3 & 2.506 \\
& 5 & 2.525 \\
& 7 & 2.530 \\
1.0 & 9 & 2.532 \\
& 11 & 2.533 \\
& $\infty$ & 2.534 \\
& 1 & 1.081 \\
& 3 & 1.082 \\
\hline
\end{tabular}

Notice that only odd values of $k$ contribute to Eq. (36). The fact that even Fourier components do not contribute is unique to the harmonic oscillator and is not a consequence of the symmetry of the system. In Table I we give the energy of the harmonic oscillator as evaluated from Eq. (35) as a function of $k_{\max }$ and $\beta \hbar \omega$. The number of Fourier coefficients required for convergence is a function of the temperature of the system. In all cases in Table I seven coefficients were sufficient to recover $99 \%$ of the total energy. It is important

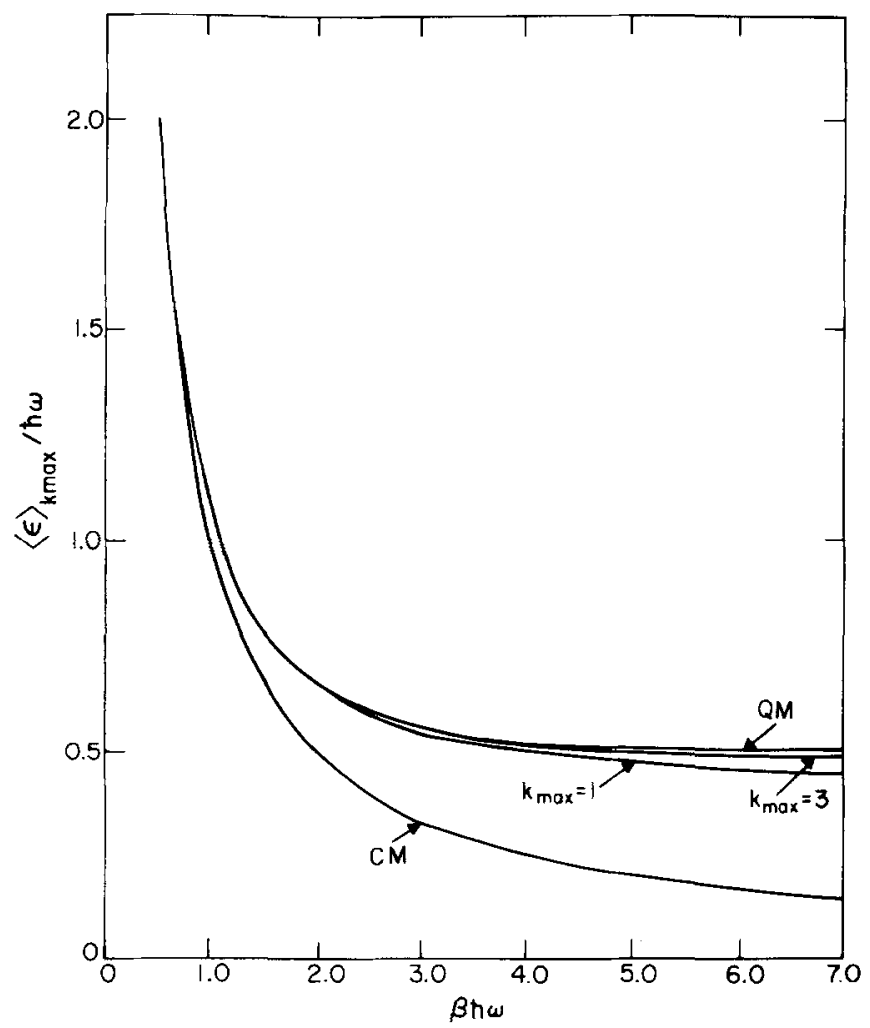

FIG. 2. The energy of a harmonic oscillator as a function of temperature evaluated with classical mechanics (CM), full quantum mechanics $(Q M)$, and from Eq. (35) with $k_{\max }=1$ and $k_{\max }=3$. to recognize that at $T=0 \mathrm{~K} \mathrm{Eq}$. (35) will not be correct for any finite $k_{\max }$. However, for low temperatures a small finite $k_{\max }$ will be adequate. To clarify this in Fig. 2 we plot $\langle\hat{\epsilon}\rangle_{k_{\max }} / \hbar \omega$ as a function of $\beta \hbar \omega$ for $k_{\max }=1,3$, and $\infty$, as well as the classical energy. As $\beta \hbar \omega$ becomes infinite all curves differ significantly from the quantum result. As $k_{\max }$ increases the temperature at which $\langle\hat{\epsilon}\rangle_{k_{\max }}$ differs significantly from the quantum result becomes lower.

To gain further insight into the utility of our approach we have also evaluated Eq. (19) numerically with Monte Carlo methods for the harmonic oscillator. A standard Metropolis-Monte Carlo algorithm was used with moves taken in the coordinates and Fourier coefficients simultaneously. The box size for the Monte Carlo moves in $a_{k}$ was taken to be $\sigma_{k}$. The results of the energy calculation are shown in Fig. 3. In Fig. 3 the solid line is the analytic result [Eq. (33)] and the points were obtained by Monte Carlo evaluation of Eq. (19). Each point on the graph was determined with one million Monte Carlo moves. The single standard deviation error bars are smaller than the resolution of the figure for this case. For the lowest temperature point $k_{\max }$ was taken to be 13, the second lowest used $k_{\max }=7$ and the remaining points all used $k_{\max }=5$. The $u$ integrations in Eq. (19) were evaluated by Gauss-Legendre quadrature with four $k_{\max }$ Gauss-Legendre points included. As a rather sensitive test of these results we also evaluated the heat capacity $C_{V}$ of the oscillator by finite difference estimates using the calculated energy points. The heat capacity is given in Fig. 4 where the solid line is the analytic result and the points include an estimate of the error bars. Agreement with the analytic result is found

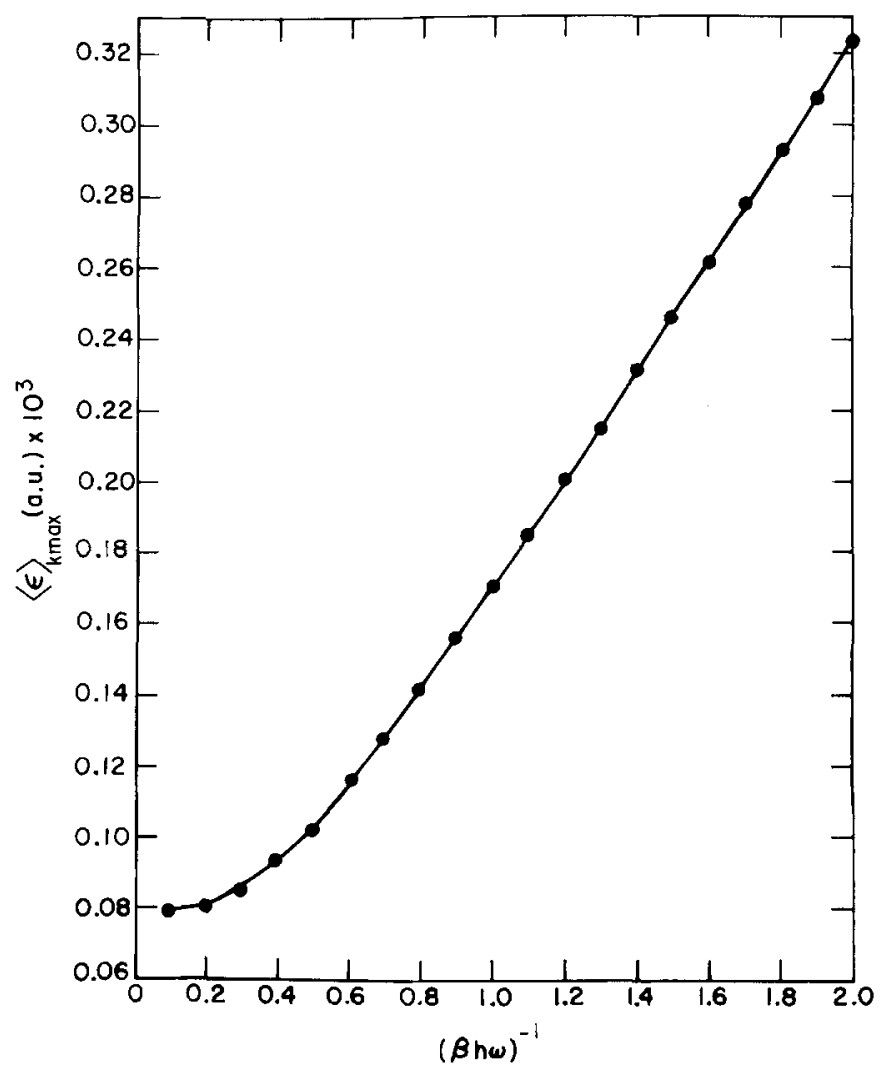

FIG. 3. The energy of a harmonic oscillator calculated by Monte Carlo evaluation of Eq. (19). The solid line is the exact result [Eq. (33)]. 


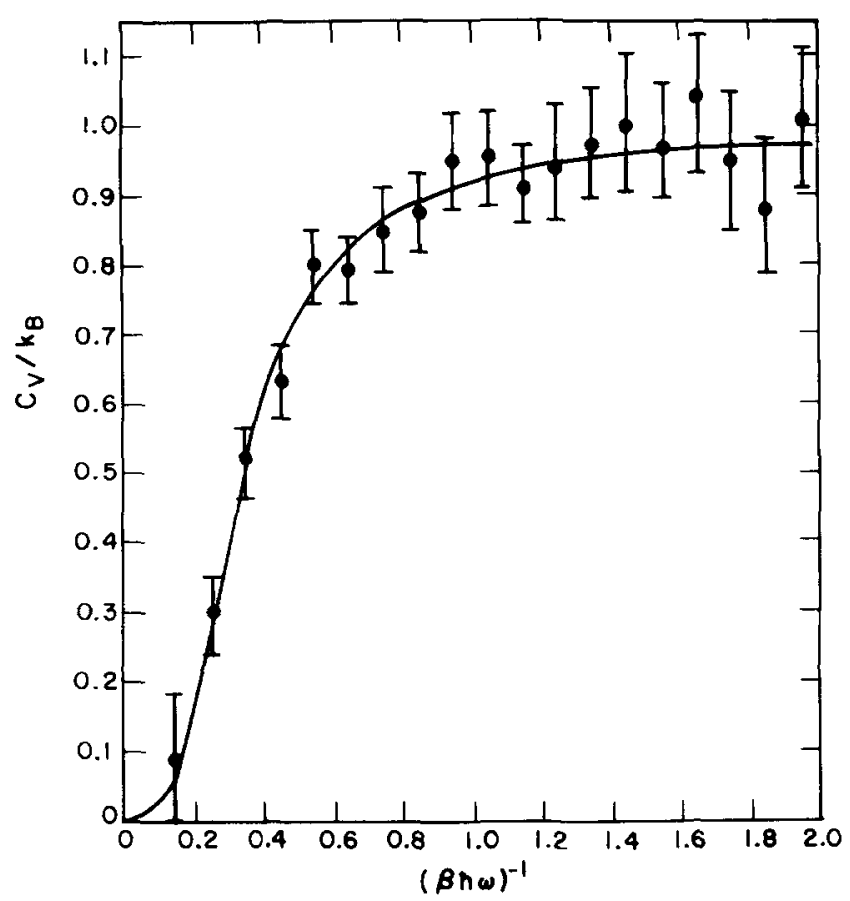

FIG. 4. The heat capacity of the harmonic oscillator evaluated by finite difference methods from the results depicted in Fig. 3. The solid line is the exact result.

over the entire calculated temperature range.

\section{B. The double-well potential}

In our previous note ${ }^{8}$ we presented energy calculations for the double-well system defined by the potential energy function

$$
V(x)=\frac{1}{2} m \omega^{2} x^{2}+A /\left[1+(x / \alpha)^{2}\right] .
$$

We repeat these results here for completeness and to extend our previous discussion. The potential function defined in Eq. (37) was chosen to assess the utility of the method for systems with strong tunneling contributions. To approximately mimic the behavior of hydrogen bonded systems we took $m$ to be the mass of a hydrogen atom and $\omega=0.006$, $A=0.009$ and $\alpha=0.09$ all in atomic units. The double well with these parameters is shown in Fig. 5. For this system the Schrödinger equation can be solved numerically, and the first five energy levels are also shown in Fig. 5 as horizontal lines. Only the ground state of this system lies below the potential barrier, and the excitation energy to the first excited state is 0.0041 a.u. The large splitting between the ground and first excited state is indicative of a system with a very high tunneling probability. Consequently quantum effects can be expected to be very large in this system. In Fig. 6 we present the internal energy of the double well as a function of temperature in units of $k_{B} / \hbar \omega$. The dashed line is the classical energy and was obtained by classical Monte Carlo techniques. The solid line was obtained by evaluation of Eq. (3) in energy representation with the 14 lowest energy states included. The change in the internal energy when 13 rather than 14 energy states were included was less than the resolution of the figure over the calculated temperature range. The solid points were obtained by Monte Carlo evaluation of $\mathrm{Eq}$. (19) with $k_{\max }$ set to 5 . Each calculated point was evaluated

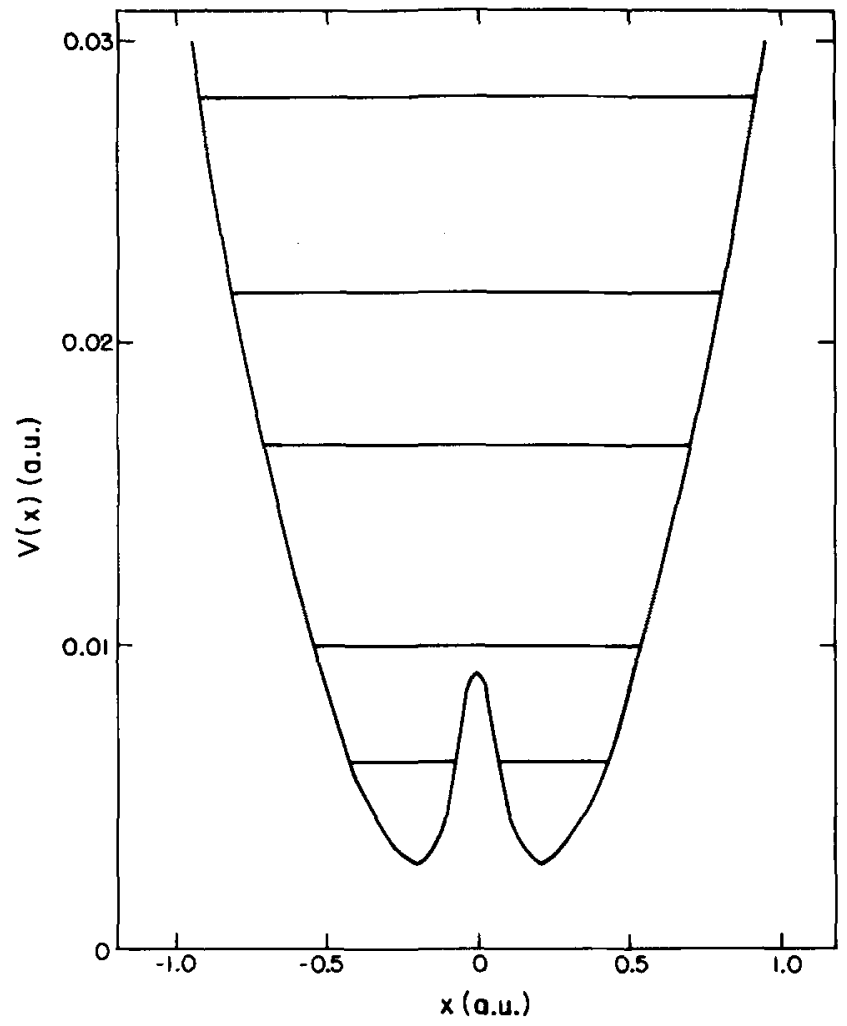

FIG. 5. The double-well potential and numerically determined energy leveis.

using one million Monte Carlo moves. As with the harmonic oscillator the $u$ integrations were evaluated with Gauss-Le-

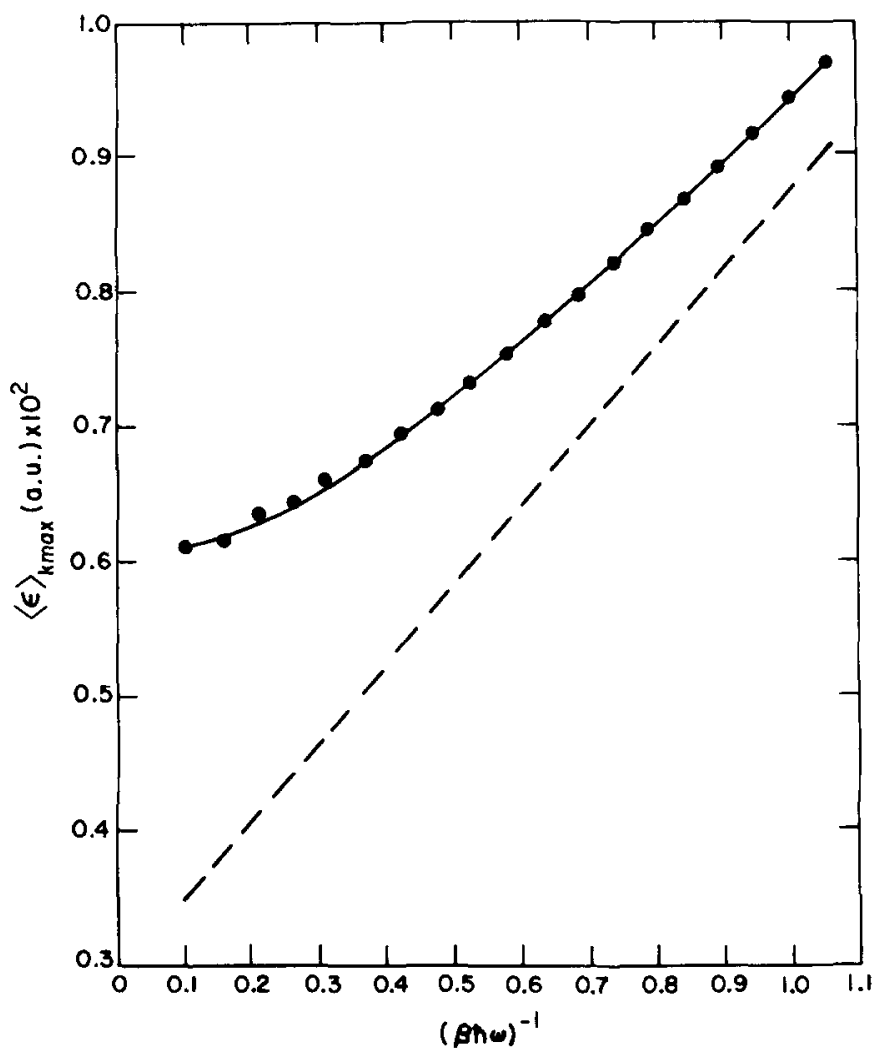

FIG. 6. The energy of the double-well potential calculated by Monte Carlo evaluation of Eq. (19). The solid line was obtained from Eq. (3) in energy representation by summation over the lowest 14 energy levels. The dashed line is the classical result. 
gendre quadrature, and moves in $x$ and $a_{k}$ were made simultaneously. The error bars associated with the calculated points are smaller than the resolution of the figure. The gap between the classical and quantum energies is very large for this system and was as large as a factor of two at the lowest calculated temperature. The Monte Carlo procedure for determining quantum mechanical properties is seen to be very successful. It is important to recognize that the numerical effort in these calculations is within an order of magnitude of the corresponding classical calculation.

\section{Cluster systems}

The thermodynamic properties of clusters is of great importance to nucleation phenomena. ${ }^{13}$ Of particular interest is the free energy of clusters as a function of the number of constituent atoms which can be directly related to steadystate nucleation rates. The calculation of the free energy of formation of atomic and molecular clusters will be the subject of future publications. In this work we use clusters as examples of interacting many particle systems to assess the utility of the methods developed in Sec. II. Cluster systems provide a sensitive test of our procedure because they incorporate realistic intermolecular forces with rotational motion in a many-body system. For the clusters considered in the present work we consider aggregates of identical atoms interacting with pairwise additive Lennard-Jones interactions; i.e.,

$$
V\left(\mathbf{r}_{1}, \ldots, \mathbf{r}_{n}\right)=\sum_{i<j}^{n} v\left(r_{i j}\right)+\sum_{i=1}^{n} v_{1}\left(\mathbf{r}_{i}\right)
$$

with

$$
v(r)=4 \epsilon\left[\left(\frac{\sigma}{r}\right)^{12}-\left(\frac{\sigma}{r}\right)^{6}\right]
$$

and

$$
v_{1}(\mathbf{r})=\left(\frac{\left|\mathbf{r}-\mathbf{R}_{\mathrm{CM}}\right|}{R_{\mathrm{C}}}\right)^{20} .
$$

In Eq. (39) $\epsilon$ and $\sigma$ are the standard Lennard-Jones parameters, and $v_{1}(\mathbf{r})$ in Eq. (38) and defined in Eq. (40) is a constraining potential. In Eq. (40) $\mathbf{R}_{\mathrm{cm}}$ is the coordinate of the center of mass of the cluster defined by

$$
\mathbf{R}_{\mathrm{cm}}=\frac{1}{n} \sum_{i=1}^{n} \mathbf{r}_{i}
$$

and $\boldsymbol{R}_{C}$ is an external parameter. In practice $\boldsymbol{R}_{C}$ is chosen so that $v_{1}(\mathbf{r})$ is very small for associated clusters and acts to reflect atoms for clusters that dissociate. At very low temperatures $v_{1}(\mathbf{r})$ is always unimportant. The potential $v_{1}(\mathbf{r})$ acts in a way which is analogous to the hard constraining wall used by Lee, Barker, and Abraham. ${ }^{14} \mathrm{~A}$ fuller discussion of the constraining potential used in this work will be given in future publications on nucleation studies. For our present purposes it is best to take Eqs. (38)-(40) as a definition of a particular interacting many-body system. In all the systems discussed here $\epsilon=119 \mathrm{~K}, \sigma=6.436673$ a.u., and $R_{C}=4 \sigma$. The Lennard-Jones parameters are appropriate for argon.
TABLE II. The internal energy of a diatomic Lennard-Jones system at $T=10 \mathrm{~K}$ as a function of the number of Fourier coefficients using Eq. (19).

\begin{tabular}{ccc}
\hline \multicolumn{1}{c}{$k_{\max }$} & $N$ & $\langle\hat{\boldsymbol{\epsilon}}\rangle_{k_{\max }} \times 10^{4 \mathrm{a}}$ \\
\hline 1 & $10^{5}$ & $-3.167 \pm 0.009$ \\
2 & $10^{6}$ & $-2.946 \pm 0.006$ \\
3 & $4 \times 10^{6}$ & $-2.843 \pm 0.001$ \\
5 & $10^{6}$ & $-2.647 \pm 0.008$ \\
8 & $10^{6}$ & $-2.625 \pm 0.006$ \\
$\langle U\rangle_{\mathrm{CM}^{*}}=(-2.636 \pm 0.003) \times 10^{-4}$ & $\langle U\rangle_{\text {est. }}=-2.36 \times 10^{-4}$ \\
\hline
\end{tabular}

Atomic units

\section{Diatomic clusters}

We first consider the Monte Carlo evaluation of the quantum mechanical internal energy of diatomic clusters. At temperatures low enough for only the ground vibrational state of this system to be occupied but large compared to the rotational temperature of the system the internal energy can be estimated as that of a classical rigid rotator and a quantum harmonic oscillator. For Lennard-Jones systems the natural frequency of vibration is given by

$$
\begin{aligned}
\omega & =\left[V^{\prime \prime}(r) / m\right]^{1 / 2} \mid r=r_{\text {eq }} \\
& =\left[57.14643812 \epsilon / m \sigma^{2}\right]^{1 / 2},
\end{aligned}
$$

where $r_{\mathrm{eq}}$ is the value of $r$ at which $v(r)$ is a minimum. For the argon system we can expect that an estimate of the internal energy will be given by

$$
\langle U\rangle_{\text {est }}=-\epsilon+\frac{\hbar \omega}{2}+\frac{\hbar \omega \exp \{-\beta \hbar \omega\}}{1-\exp \{-\beta \hbar \omega\}}+5 / 2 \beta,
$$

which gives $-2.36 \times 10^{-4}$ a.u. at $10 \mathrm{~K}$. In Table II we give $\langle\hat{\epsilon}\rangle_{k_{\max }}$ as evaluated with Eq. (19) as a function of $k_{\max }$. For comparison the classical energy of this system is $(-2.636 \pm 0.003) \times 10^{-4}$ a.u. calculated by Monte Carlo integration. Notice that the quantum estimate of the internal energy bounds the classical energy from above as it must. In Table II, $N$ is the number of Monte Carlo passes included in the evaluation of Eq. (19). In contrast to the quantum estimate Eq. (19) predicts energies which bound the classical result from below which is impossible. These results indicate that Eq. (19) converges very slowly with respect to the number of Fourier coefficients included. The slow convergence is a consequence of the fact that the virial theorem is valid only for the trace over the exact density matrix. Evaluation of the trace of the virial operator over approximate density operators may be significantly in error as in Eq. (19). In Table III we give the energy of the diatomic cluster calculated from Eq. (16) as a function of $k_{\max }$. One million Monte Carlo points were used for each energy given in Table III. Unlike the virial expansion [Eq. (19)] the direct evaluation of the energy [Eq. (16)] converges very rapidly with respect to the number of Fourier coefficients and the results agree well with the estimate obtained from Eq. (44). The differences between Eq. (44) and the results in Table III are due to anharmonicity corrections, rotational-vibrational coupling, and quantum corrections to the rotational energy.

The origin of the poor convergence for this system when the virial expressions are used can be understood by examining the virial operator 


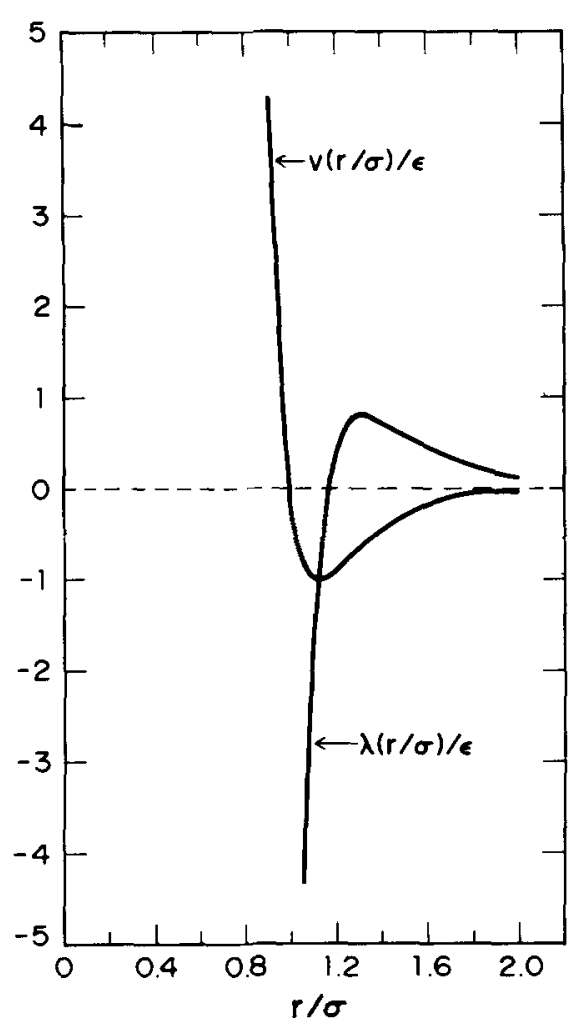

FIG. 7. The Lennard-Jones potential $v(r)$ and associated virial operator $\lambda(r)$.

$$
\hat{\epsilon}\left(\mathbf{r}_{1}, \ldots, \mathbf{r}_{n}\right)=11 \sum_{i=1}^{n}\left(\left|\mathbf{r}_{i}-\mathbf{R}_{\mathrm{cm}}\right| / R_{C}\right)^{20}+\sum_{i<j}^{n} \lambda\left(r_{i j}\right),
$$

where

$$
\lambda(r)=4 \epsilon\left[2\left(\frac{\sigma}{r}\right)^{6}-5\left(\frac{\sigma}{r}\right)^{12}\right] .
$$

In Fig. $7 \lambda(r)$ is plotted along with $v(r)$ for comparison. For $r<r_{\text {eq }}$, where $r_{\text {eq }}$ is the value of $r$ at which $v(r)$ is minimum, $\lambda(r)$ becomes steeply negative. With quantum mechanical wanderings introduced by nonzero Fourier coefficients the deep negative values of $\lambda(r)$ greatly contribute to $\langle\hat{\epsilon}\rangle_{k_{\max }}$. Only for very large $k_{\max }$ will the average coordinate contributing to $\langle\hat{\epsilon}\rangle_{k_{\max }}$ exceed $r_{\text {eq }}$ sufficiently to make Eq. (19) well converged.

The poor convergence found in Eq. (19) can be expected for systems with potentials with large short-range repulsions. It is important to emphasize that the convergence of the density operator with $k_{\max }$ is excellent for systems with strong short-range repulsions. This is made clear in Table III. The errors are incurred by use of the virial theorem for strongly repulsive systems. For Lennard-Jones systems excellent results will be obtained from Eq. (16), and Eq. (19) is to be avoided.

TABLE III. The internal energy of a diatomic Lennard-Jones system at $T=10 \mathrm{~K}$ as a function of the number of Fourier coefficients using Eq. (16).

\begin{tabular}{cc}
\hline$k_{\max }$ & $\langle\hat{H}\rangle_{k_{\max }} \times 10^{43}$ \\
\hline 1 & $-2.333 \pm 0.003$ \\
2 & $-2.332 \pm 0.005$ \\
$\langle U\rangle_{\mathrm{CM}^{*}}=(-2.636 \pm 0.003) \times 10^{-4}\langle U\rangle_{\mathrm{est}^{4}}=-2.36 \times 10^{-4}$ \\
\hline
\end{tabular}

Atomic units.
TABLE IV. The total internal energy of a 13 particle cluster of argon atoms at $T=10 \mathrm{~K}[\mathrm{Eq} .(16)]$.

\begin{tabular}{cc}
\hline \hline$k_{\max }$ & $\langle\hat{H}\rangle_{k_{\max }{ }^{a}}$ \\
\hline 1 & $(-0.1411 \pm 0.0001) \times 10^{-1}$ \\
2 & $(-0.1413 \pm 0.0002) \times 10^{-1}$ \\
$\langle U\rangle_{\mathrm{CM}^{3}}=(-0.15580 \pm 0.00003) \times 10^{-1}$ \\
\hline
\end{tabular}

atomic units.

\section{Polyatomic clusters}

To assess the applicability of the technique developed in Sec. II to interacting many-particle systems we have used the method to calculate the thermodynamic properties of polyatomic clusters of Lennard-Jones atoms. As in the diatomic case we have chosen the Lennard-Jones parameters to be appropriate for argon. In Table IV we give the internal energy of a 13 particle cluster of argon atoms evaluated with Eq. (16) at $T=10 \mathrm{~K}$ as a function of $k_{\max }$. Each calculation consisted of 20000 Monte Carlo passes used to initialize the system followed by 50000 Monte Carlo passes during which data was accumulated. In each Monte Carlo pass the individual atoms were moved separately using the algorithm of Metropolis et al. ${ }^{10}$ The Fourier coefficients were altered simultaneously with the particles using $\sigma_{k i}$ as a box size. Consequently a total of 650000 Monte Carlo points were used in the evaluation of $\langle U\rangle$ in Table IV. The classical internal energy is also given in Table IV, and 100000 Monte Carlo passes were used in its evaluation. From Table IV we see that only one Fourier coefficient is required for the quantum energy to converge to four figures at $10 \mathrm{~K}$. The quantum correction to the energy is on the order of $10 \%$ at this temperature. For the $k_{\max }=1$ calculations the computer time required was a factor of 4.6 times longer than the classical calculation. In the evaluation of Eq. (16) the $u$ integrations were performed with four $k_{\max }$ Gauss-Legendre points. Experiments with larger numbers of Gauss points resulted in no change in the energy to the accuracy we report in Table IV.

In Table $V$ we present the ratio of the quantum to classical partition functions $\Delta$ as calculated from $\mathrm{Eq}$. (32) as a function of temperature for a 13 particle cluster. In each calculation $k_{\max }$ was set to 4 and data was accumulated with 20000 Monte Carlo passes. In Table VI we present $\Delta$ as a function of the cluster size $n$ calculated at $T=30 \mathrm{~K}$. For the numbers presented in Table VI, $k_{\max }$ was set to 4 and 20000 Monte Carlo passes were used. From Tables V and VI we see that quantum effects, as measured by $\Delta$, become increasingly important as the temperature is lowered and as the cluster

TABLE $V$. The ratio of the quantum to classical partition functions for a 13 particle argon cluster [ $k_{\max }$ set to 4$]$.

\begin{tabular}{cl}
\hline $\mathrm{T}(\mathrm{K})$ & \multicolumn{1}{c}{$\Delta$} \\
\hline 25 & $0.050 \pm 0.008$ \\
30 & $0.15 \pm 0.02$ \\
35 & $0.31 \pm 0.02$ \\
40 & $0.45 \pm 0.03$ \\
\hline
\end{tabular}


TABLE VI. The ratio of the quantum to classical partition functions for clusters of Ar atoms at $30 \mathrm{~K}$ [ $k_{\max }$ set to 4].

\begin{tabular}{cc}
\hline \hline$n$ & $\underline{\Delta}$ \\
\hline 10 & $0.31 \pm 0.02$ \\
11 & $0.29 \pm 0.02$ \\
12 & $0.20 \pm 0.02$ \\
13 & $0.15 \pm 0.02$ \\
14 & $0.13 \pm 0.02$ \\
15 & $0.12 \pm 0.02$ \\
16 & $0.11 \pm 0.02$ \\
17 & $0.09 \pm 0.01$ \\
\hline
\end{tabular}

size increases. The inverse relation between $\Delta$ and $n$ arises because as $n$ increases the number of the vibrational modes increases, whereas the importance of the classical rotational and translational modes remain effectively unchanged.

\section{CONCLUSIONS}

Using Fourier representations of Feynman path integrals we have developed and implemented a practical scheme for quantum mechanical calculations of the thermodynamic properties of complex interacting many-particle systems. Our method utilizes the Monte Carlo algorithm developed by Metropolis et al ${ }^{10}$ for classical systems and requires computational effort within an order of magnitude of the corresponding classical calculation. We have found that the expansion of the path integrals in a Fourier series converges rapidly for a variety of systems over a wide temperature range. Unlike other related approaches to quantum statistical mechanics ${ }^{2-5,7}$ our approach requires no special treatment for the important class of systems with potentials having strongly repulsive cores. We have applied the method to one-dimensional problems with analytic or numerically exact results for comparison. We have also applied the method to the calculation of the thermodynamic properties of clusters of Lennard-Jones atoms. In all cases the results have been very accurate.

Although the method we have developed can be formally applied to systems where quantum particle statistics are important the numerical requirements are presently limited to systems obeying Boltzmann statistics. Methods for extending the approach to cases where particle statistics are important are under study.

We are presently applying the technique to evaluate fully quantum mechanical expressions for the free energy of formation of clusters and nucleation rates. The results of the cluster calculations will appear separately.

\section{ACKNOWLEDGMENTS}

We wish to thank J. Winterkamp of Los Alamos National Laboratory's Group ESS-5 for computational support. One of us (DLF) wishes to thank the Chemistry Division at Los Alamos National Laboratory for hospitality and support while on Sabbatical Leave from the University of Rhode Island. Work at the University of Rhode Island was supported in part by grants from Research Corporation and the University of Rhode Island Computer Center. One of us (DLF) would like to thank Professor Sze Yang for helpful discussions.

\section{APPENDIX}

In the expressions we derived in Sec. II we evaluated the path integrals which occurred in Eq. (8) as a Fourier series about a straight line path. Although this series proved to be very rapidly convergent in all cases we have studied (see Sec. III) in some systems other choices for primary paths may be more convenient. For example if our system were well approximated by harmonic motion with a natural frequency $\omega$ expansion about a classical harmonic oscillator path may be more convenient. This notion was also suggested by Miller. ${ }^{11}$ For the $x$ component of particle $i$ the expansion of paths would take the form

$$
\begin{aligned}
x_{i}(u)= & x_{i} \cosh (\omega u)+\frac{\left(x_{i}^{\prime}-x_{i} \cosh (\beta \hbar \omega)\right)}{\sinh (\beta \hbar \omega)} \sinh (\omega u) \\
& +\sum_{k=1}^{\infty} a_{k x i} \sin \frac{k \pi u}{\beta \hbar} .
\end{aligned}
$$

Introduction of Eq. (A1) into Eq. (8) results in the expression

$$
\begin{aligned}
& \rho\left(\mathbf{r}_{1}, \ldots, \mathbf{r}_{N} ; \mathbf{r}_{1}^{\prime}, \ldots, \mathbf{r}_{N}^{\prime}\right) \\
& \quad=J^{\prime} \exp \left\{-\sum_{i=1}^{N} \frac{m_{i} \omega}{2 \hbar \sinh (\beta \hbar \omega)}\left[\left(r_{i}^{2}+r_{1}^{\prime 2}\right) \cosh (\beta \hbar \omega)\right.\right. \\
& \left.-2 \mathbf{r}_{i} \cdot \mathbf{r}_{i}^{\prime}\right] \int \prod_{k=1}^{\infty} d^{3} a_{k 1} \cdots d^{3} a_{k N} \exp \left\{\sum_{i=1}^{N} a_{k i}^{2} / 2 S_{k i}^{2}\right\} \\
& \quad \times \exp \left\{-\frac{1}{\hbar} \int_{0}^{\beta \hbar}\left(V\left[\mathbf{r}_{1}(u), \ldots, \mathbf{r}_{N}(u)\right]\right.\right. \\
& \left.\left.-V_{0}\left[r_{1}(u), \ldots, r_{N}(u)\right]\right) d u\right\},
\end{aligned}
$$

where $J^{\prime}$ is a Jacobian factor independent of $\mathbf{r}_{i}$ and $V$ for all $i$;

$$
S_{k i}=\left[2 \beta \hbar^{2} /\left(m_{i}(\pi k)^{2}+m_{i}(\beta \hbar \omega)^{2}\right)\right]^{1 / 2}
$$

and $V_{0}\left(\mathbf{r}_{1}, \cdots, \mathbf{r}_{N}\right)$ is the oscillator potential

$$
V_{0}\left(\mathbf{r}_{1}, \cdots, \mathbf{r}_{N}\right)=\sum_{i=1}^{N} \frac{1}{2} m_{i} \omega^{2} r_{i}^{2}
$$

The oscillator reference has the appealing feature that the Gaussian width [Eq. (A3)] vanishes both for small and large $\beta$. In practice Eq. (A2) has not proved to be more rapidly convergent than the straight line parametrization for those cases we have studied.

'J. P. Valleau and S. G. Whittington, in Statistical Mechanics, edited by B.

J. Berne (Plenum, New York, 1977), p. 137.

${ }^{2}$ J. A. Barker, J. Chem. Phys. 70, 2914 (1979).

${ }^{3}$ D. Chandler and P. G. Wolynes, J. Chem. Phys. 74, 4078 (1981).

${ }^{4}$ K. S. Schweizer, R. M. Stratt, D. Chandler, and P. G. Wolynes, J. Chem. Phys. 75, 1347 (1981).

${ }^{5}$ R. M. Stratt, J. Chem. Phys. 77, 2108 (1982).

${ }^{6} J$. D. Doll and L. E. Myers, J. Chem. Phys. 71, 2880 (1979).

${ }^{7}$ D. Thirumalai, E. J. Bruskin, and B. J. Berne, J. Chem. Phys. 79, 5063 (1983).

${ }^{B}$ J. D. Doll and D. L. Freeman, J. Chem. Phys. 80, 2239 (1984).

${ }^{9}$ R. P. Feynman, Statistical Mechanics (Benjamin, Reading, Mass., 1972).

${ }^{10}$ M. Metropolis, A. W. Rosenbluth, M. H. Rosenbluth, A. H. Teller, and E. Teller, J. Chem. Phys. 21, 1087 (1953).

"W. H. Miller, J. Chem. Phys. 63, 1166 (1975).

${ }^{12} \mathrm{~J}$. P. Valleau and G. M. Torrie, in Statistical Mechanics, edited by B. J. Bern, (Plenum, New York, 1977), p. 169.

${ }^{13}$ F. F. Abraham, Homogeneous Nucleation Theory (Academic, New York, 1977).

${ }^{14}$ J. K. Lee, J. A. Barker, and F. F. Abraham, J. Chem. Phys. 58, 3166 (1973). 